\title{
Identification of novel Mlo family members in wheat and their genetic characterization
}

\author{
Shogo Konishi ${ }^{1,2}$, Tetsuo Sasakuma ${ }^{1}$ and Tsuneo Sasanuma ${ }^{1,2 *}$ \\ ${ }^{1}$ Kihara Institute for Biological Research, Yokohama City University, Maioka-cho 641-12, \\ Totsuka-ku, Yokohama 244-0813, Japan \\ ${ }^{2}$ Faculty of Agriculture, Yamagata University, Wakaba-machi 1-23, Tsuruoka, \\ Yamagata 997-8555, Japan
}

(Received 21 June 2010, accepted 31 August 2010)

\begin{abstract}
Mlo is a plant-specific gene family, which is known to show stress responses in various plants. To reveal the genetic characteristics of the Mlo family in wheat, we isolated wheat Mlo members from a database and studied their expression in young shoots and roots under salt and osmotic stress conditions. In an in silico investigation, we identified seven Mlo members in wheat and named them TaMlo1 TaMlo-7. None of the wheat Mlo showed significant induction or reduction of their expression under salt or osmotic stress, but organ-specific expression was observed in several TaMlo members. TaMlo-1, TaMlo-2, and TaMlo-5 were constitutively expressed in both shoots and roots, but TaMlo-3 and TaMlo-4 showed root-specific expression, and TaMlo-7 showed dominant expression in shoots. TaMlo- 6 was weakly expressed in both shoots and roots. Phylogenetic analysis classified the plant Mlo members into six classes; four of them were comprised of angiosperm Mlo members, and the remaining two consisted of fern and moss Mlo members. The seven wheat Mlo members were classified into four angiosperm Mlo classes, similar to those of Arabidopsis and rice, indicating that the formation of each of the Mlo classes preceded the divergence of dicots and monocots. The differentiation of the expressional patterns among the seven TaMlo members was not related to their phylogenetic classification. This result suggested that the organ specific expression of individual Mlo members occurred relatively recently in their evolution.
\end{abstract}

Key words: Mlo, wheat (Triticum aestivum), gene family, RT-PCR, stress response

\section{INTRODUCTION}

The Mlo genes encode a plant-specific and sequencediversified class of seven transmembrane (7-TM) proteins that form a multigene family in both monocot and dicot plants (Büschges et al., 1997; Devoto et al., 1999). To date, many $M l o$ homologs have been identified in various plants. The families of two model plants, Arabidopsis thaliana (Devoto et al., 2003) and rice (Liu and Zhu, 2008), whose whole genome sequences have been determined, contain 15 and 12 members, respectively. Liu and Zhu (2008) further reported that Mlo members can be divided into four groups based on their phylogenetic relationships.

With respect to function, Mlo was first defined as the

\footnotetext{
Edited by Takashi Endo

* Corresponding author. E-mail: sasanuma@tds1.tr.yamagata-u. ac.jp
}

locus controlling disease resistance to powdery mildew in barley (Jørgensen, 1992; Wolter et al., 1993). Homozygotes for the recessive allele ( $m l o$ ) show a wide spectrum of resistance to the powdery mildew fungus, Blumeria graminis f.sp. hordei (Jørgensen, 1992). Expressional analyses in barley found that Mlo transcripts accumulate in response to infection with the fungus and that overexpression of Mlo results in supersusceptibility to the fungus (Wolter et al., 1993; Kim et al., 2002; Piffanelli et al., 2002). The accumulation of the mlo transcript was also detected in rice infected with blast fungi and in wheat injected with powdery mildew-derived carbohydrate (Piffanelli et al., 2002). Recently, using Mlo mutant alleles in barley, Reinstädler et al. (2010) revealed the regions of the MLO protein that are functionally important for resistance to powdery mildew. However, microarray analyses found that not only the Mlo genes but also more than 300 other genes showed expressional changes after powdery mildew infection in barley, and some of them were 
induced or repressed by infection with other pathogens, such as rust and blast, suggesting that the powdery mildew resistance induced by the Mlo genes is part of a complex response to various pathogens (Zellerhoff et al., 2010). The expression of Mlo was also increased during leaf senescence, by wounding, and by Paraquat treatment ((Piffanelli et al., 2002). These findings suggest that Mlo is likely to have a functional role in cell death protection during periods of biotic and abiotic stress (Wolter et al., 1993; Peterhänsel et al., 1997; Piffanelli et al., 2002). Less information is available about the function of the Mlo genes under abiotic stress compared with the amount of data available about their role under biotic stress, but the involvement of the Mlo genes in abiotic stress has been investigated in previous reports. Microarray profiling of NaCl-treated Arabidopsis roots showed that five of the 14 Mlo genes were up- or downregulated together with several other signal transduction genes (Jiang and Deyholos, 2006). Although the multiple response functions of Mlo were indicated in previous studies, the correspondence between their functional differentiation and subfamily diversification has not been clarified.

In wheat, three homoeologous cDNA of Mlo (TaMlo-1A, $-1 B$ and $-1 D$ ) were isolated as homologs of barley $M l o$ (Elliott et al., 2002). Recently, we found that a wheat partial cDNA (WESR3) induced by salt stress (Nemoto and Sasakuma, 2002) had $48 \%$ identity with barley $M l o$ in terms of its amino acid sequence but that its nucleotide sequence was not significantly similar to any member of TaMlo-1. This implies the presence of an additional Mlo locus in wheat. Considering the multiplicity of the Mlo genes in other plant species and the large genome size of wheat, additional members of the Mlo family should exist in wheat. Identification of more Mlo members in wheat would be helpful for understanding the evolution and functional differentiation of Mlo. Since Mlo is a key gene for resistance to powdery mildew, which is one of the most serious wheat and barley diseases, the finding of new Mlo members would contribute to wheat and barley breeding. In addition, although various sequences of $M l o$ have been identified in barley, they were allelic to each other; in other words, the barley Mlo sequences reported belonged to one class of the Mlo gene family (Piffanelli et al., 2004, Tacconi et al., 2006, Liu and Zhu, 2008). Therefore, the discovery of new Mlo members in wheat would contribute to the study of Mlo in barley.

In this study, we performed an in silico search for Mlo members in wheat followed by PCR amplification using sequence information for the Mlo members of other plants. For all Mlo candidates, we conducted expressional analysis in various tissues under abiotic stress. Based on these results, the structural and functional differentiation of the Mlo gene family in wheat was discussed.

\section{MATERIALS AND METHODS}

in silico search for Mlo homologs We searched for wheat Mlo homologs in two DNA databases, Wheat Gene Index ver. 11.0 (http://www.tigr.org) and GenBank (http:/ /ncbi.nlm.nih), with the computer programs BLASTN and TBLASTN using published rice and Arabidopsis Mlo sequences (Devoto et al., 1999, Chen et al., 2006, Liu and Zhu, 2008) as queries. The BLAST search was performed with the following criterion: E-value $<1 \mathrm{e}-10$. The obtained wheat $M l o$ homolog candidates were aligned and assembled into unified sequences with the software CAP3 (Huang and Madan, 1999). Then, the transmembrane domains of the protein sequences deduced from the unified sequences were predicted by HMMTOP (Tusnády and Simon, 2001) and Pepwindowall (Kyte and Doolittle, 1982). The presence of the in silico deduced unified sequences in the wheat transcriptome was verified by sequencing of the RT-PCR products amplified using spe-

Table 1. Information about the seven wheat Mlo members classified in this study

\begin{tabular}{|c|c|c|c|c|c|c|c|c|}
\hline \multirow[t]{2}{*}{ Member } & \multirow[t]{2}{*}{$\begin{array}{l}\text { Number of } \\
\text { EST hits }\end{array}$} & \multicolumn{3}{|c|}{$\begin{array}{c}\text { Nucleotide length of } \\
\text { unified sequence obtained in silico }\end{array}$} & \multicolumn{3}{|c|}{$\begin{array}{l}\text { Nucleotide length of } \\
\text { the sequence determined by RT-PCR }\end{array}$} & \multirow[t]{2}{*}{$\begin{array}{l}\text { Accession } \\
\text { number }\end{array}$} \\
\hline & & 5'-UTR & CDS & 3'-UTR & 5'-UTR & CDS & 3'-UTR & \\
\hline TaMlo- $1^{\mathrm{a}}$ & 26 & 197 & 1605 & 84 & n.d. & n.d. & n.d. & AX063298 \\
\hline TaMlo-2 & 12 & 165 & 1497 & 198 & 18 & 1497 & 148 & AB581575 \\
\hline TaMlo- $3^{\mathrm{b}}$ & 10 & 43 & 787 & - & - & 615 & - & AB581579 \\
\hline TaMlo-4 & 11 & - & 1497 & 120 & - & 1497 & 56 & AB581576 \\
\hline TaMlo-5 & 28 & 25 & 1476 & 165 & 3 & 1476 & 58 & AB581580 \\
\hline TaMlo-6 & 4 & 169 & 1683 & 296 & 33 & 1683 & 112 & AB581577 \\
\hline TaMlo-7 & 11 & 127 & 1494 & 328 & 91 & 1494 & 220 & AB581578 \\
\hline
\end{tabular}

a The nucleotide length and accession number of TaMlo-1 indicate those of TaMlo-1A reported in Elliott et al. (2002). n.d.: not determined in this study.

b TaMlo-3 was a partial sequence containing only the upstream half of the coding region. 
TM1

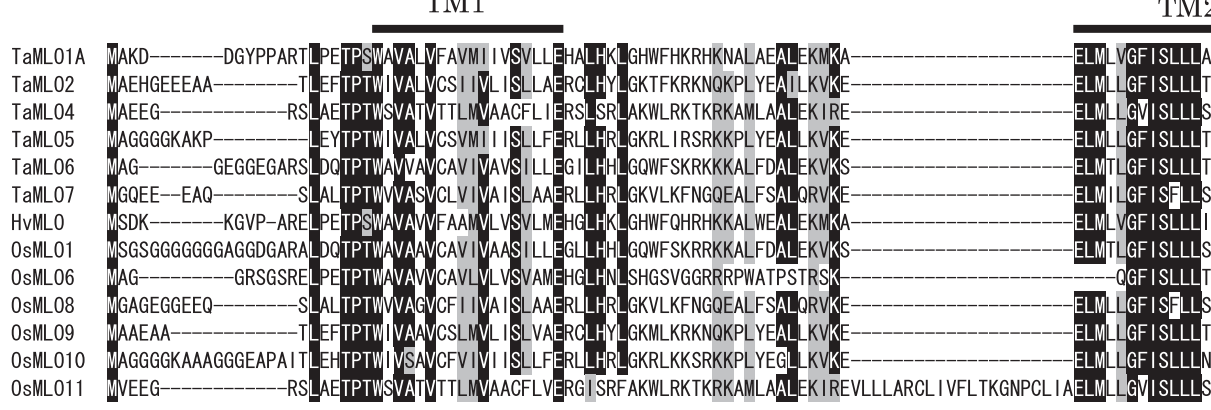

TM2

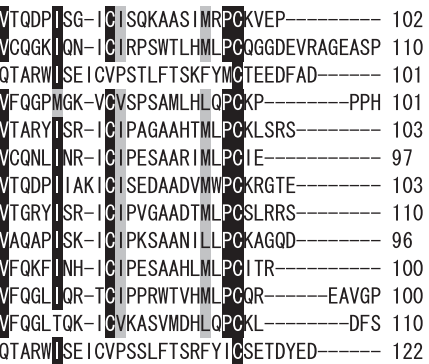
TM3

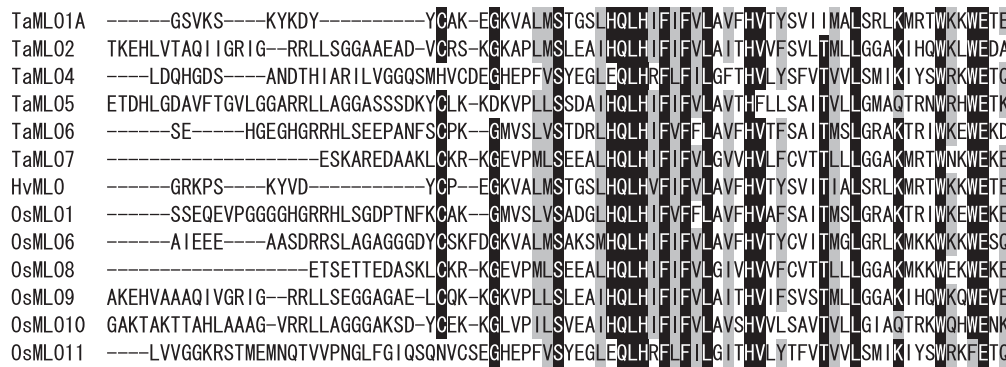

OsML011 ---LVVGGKRSTMEMNQTVVPNGLFGI QSQNVCSEGHEPFVSYEGLEQLHRFLFILG ITL

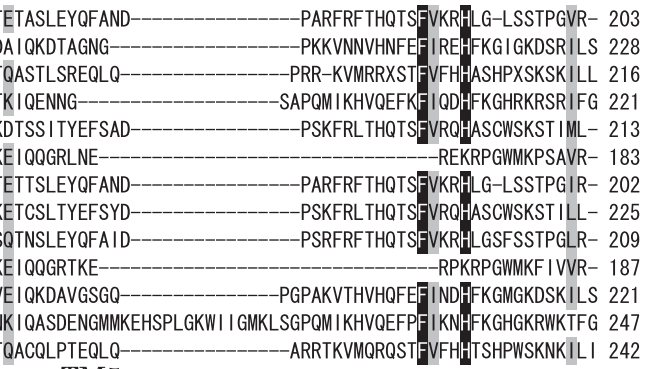

TM5

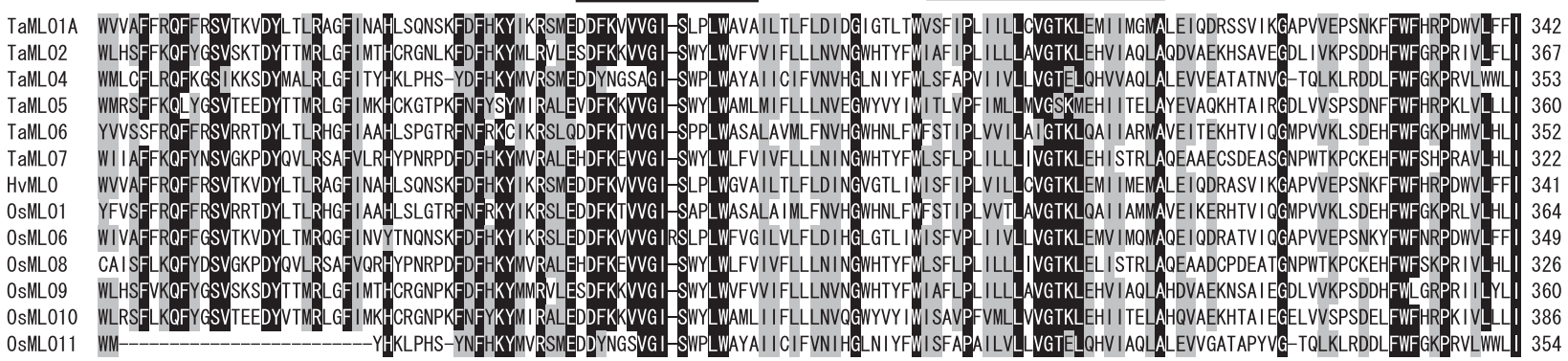

TM6

TM7

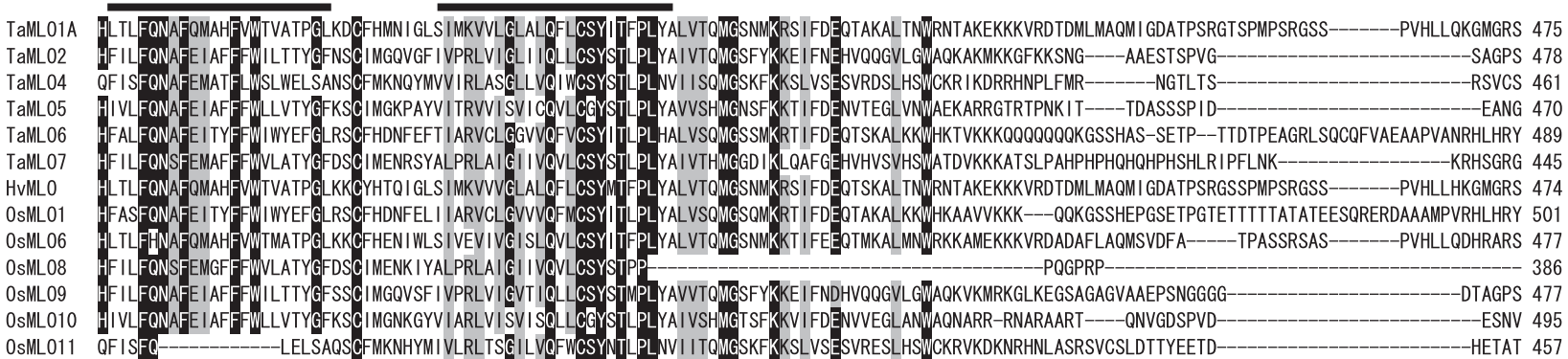

TaML01A DDPQSAPTSPRTMEEARDMYPVVVAHPVHRLN--PADRRRSVSSSALDADIPSAD--_-_----FSFSQG----_--- 534

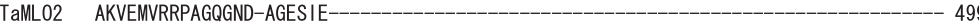

TaML04 LGTLSRTVSASSLYYALTVVTVDD---EEEISHIEQDIQHP-_-_-_-_-_-_-_-_-_-_-_-_-_-_-_-_-_-_ 499

TaML05 GAVQMTNTRANSSVEQGTARLI-_-_-_ 492

TaML06 KT IAHVGATRALSDSDCSDTDA--EAQTRYLIPPTKQRNLDS---EVRVDVDAAT----PDHRDSFSFQKPPPAQNAMEK 560

TaML07 PATEEAAAEARATEORAGSSSTPTAPPOORADDLEEIVTTTEEDHRRRNASFS-_-_-_-_-_-_-_-_ 498

HvMLO DDPQSAPTSPRTQQEARDMYPVVVAHPVHRLN--PNDRRRSASSSALEADIPSAD--_-_----FSFSQG---_-_-- 533

0sML01 KTIAHVGATGTLSDSDCSDTDTPFASPTRLLIPPTKQRSLDAGRAEVRVDVDSTPTPTPPERHDSFSFPR-LPAHNLQOK 580

0sML06 DDPPSPI TVASPPAPEEDIYPVPAAAASRQLLDDPPDRRWMASSS---ADIADSD---_----FSFSAQR---_-- 536

0sML08 -

0sMLO09 VKIEMMRRAAREGNDEAGVSIE--

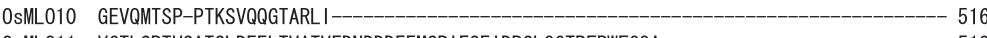

0sML011 VGTLSRTVSATSLDEELTVATVEDNDDDEEMSRI EQE IDRSLGGTRFPWFCOA-_-_-_-_-_-_-_-_-_-_- 510

Fig. 1. Multiple alignment of the deduced amino acid sequences of the 13 Mlo members from wheat, barley, and rice. Although rice has 12 Mlo members (Liu and Zhu, 2008), five members were selected to represent the phylogenetically divergent groups of the Mlo gene family. The alignment was conducted by ClustalW. Consensus matches are indicated by darker shading for identities and light shading for conservative substitutions. The seven putative transmembrane domains of TaMLO-1A (TM1 TM7) predicted by HMMTOP are indicated by black bars above the sequences. 
cific primers for each Mlo member.

Expressional analysis Seeds of Triticum aestivum L. cv. Chinese Spring (accession number KT020-003) provided by National BioResource Project (NBRP) KOMUGI were sterilized in $\mathrm{NaClO}$ solution (2.0\% effective chloride) and sown on Murashige-Skoog (MS) medium (1\% agar, supplemented with $150 \mathrm{mM} \mathrm{NaCl}$ or $2.5 \%$ mannitol) in sterilized glass bottles. The seedlings in the bottles were grown in a growth chamber under the following conditions: $20^{\circ} \mathrm{C}, 50 \%$ humidity, and $18 \mathrm{~h}$ illumination with fluorescent lights $\left(170-240 \mathrm{mmolm}^{-2} \mathrm{~s}^{-1}\right)$. Total RNA was extracted from two-week-old roots and shoots with TRIzol reagent. The first-strand cDNA was synthesized from 10 $\mu \mathrm{g}$ total RNA using oligo dT15 primers and the reverse transcriptase SuperScript III (Invitrogen). RT-PCR was carried out using $10 \mathrm{pmol}$ of each of the primers for the wheat Mlo members and the $\alpha$-tubulin gene in a $20 \mu \mathrm{l}$ reaction mixture containing 0.5 units of a Takara Taq polymerase. The sequences of the member-specific primers used for the expressional analysis were AAGTTCTTCTGGTTCCACCG and TGGCTGAAGGAAAAATCTGC for TaMlo-1, CCATCGGATGACCACTTCTG and TTGCCCCCAATGTTTTACGG for TaMlo-2, CTGCTCCTGCTGGGCTTTGG and CTCCACCTGCCAGGAGTCTC for TaMlo-3, TCCAGAATGCCTTTGAGATG and CAGTCAGCGCCTCATACAG for TaMlo-4, GGATCACCTTGGTTCCATTC and ATCATGGGGAAACCAGCATA for TaMlo-5, GTCACGGCGAGGTACATATC and GCTGCCTCACGAAAGAAGTC for TaMlo-6, and CGGCACGAGGCTAAGATGAG and CTCATCTTAGCCTCGTGCC for TaMlo-7. The PCR conditions were as follows: $5 \mathrm{~min}$ at $94^{\circ} \mathrm{C} ; 24$ cycles of $30 \mathrm{sec}$ at $94^{\circ} \mathrm{C}, 30 \mathrm{sec}$ at $60^{\circ} \mathrm{C}$, and $30 \mathrm{sec}$ at $72^{\circ} \mathrm{C}$; and $5 \mathrm{~min}$ at $72^{\circ} \mathrm{C}$. The amplified products were stained in a gel with $\mathrm{SYBR}^{\circledR}$ GOLD (Molecular probe) and were quantified based on their fluorescence intensity using an FLA5000 and the Image Gauge software (Fujifilm). The amount of $\alpha$-tubulin was used as an internal control to calculate the relative amounts of Mlo expression. Quantification by RT-PCR was conducted using three separate plants for each treatment, and the measurements were averaged.

Phylogenetic analysis Multiple alignment between

Table 2. Amino acid sequence identity matrix of TaMlo genes and their homologs in rice and barley

\begin{tabular}{|c|c|c|c|c|c|c|}
\hline Gene & TaMlo- $1^{\mathrm{a}}$ & TaMlo-2 & TaMlo-4 & TaMlo-5 & TaMlo-6 & TaMlo-7 \\
\hline \multicolumn{7}{|l|}{ within wheat } \\
\hline TaMlo-1 & - & & & & & \\
\hline TaMlo-2 & 0.328 & - & & & & \\
\hline TaMlo-4 & 0.260 & 0.317 & - & & & \\
\hline TaMlo-5 & 0.321 & 0.516 & 0.278 & - & & \\
\hline TaMlo- 6 & 0.405 & 0.342 & 0.295 & 0.304 & - & \\
\hline TaMlo-7 & 0.304 & 0.413 & 0.285 & 0.360 & 0.304 & - \\
\hline \multicolumn{7}{|c|}{ between wheat and barley } \\
\hline HvMlo & 0.851 & 0.346 & 0.268 & 0.323 & 0.415 & 0.310 \\
\hline HvMlo2 & 0.662 & 0.345 & 0.261 & 0.323 & 0.395 & 0.296 \\
\hline \multicolumn{7}{|c|}{ between wheat and rice } \\
\hline OsMlo-1 & 0.413 & 0.342 & 0.296 & 0.308 & 0.780 & 0.309 \\
\hline OsMlo-2 & 0.319 & 0.708 & 0.334 & 0.525 & 0.344 & 0.409 \\
\hline OsMlo-3 & 0.634 & 0.340 & 0.259 & 0.317 & 0.399 & 0.299 \\
\hline OsMlo-4 & 0.245 & 0.287 & 0.349 & 0.235 & 0.275 & 0.268 \\
\hline OsMlo-5 & 0.291 & 0.507 & 0.293 & 0.539 & 0.294 & 0.379 \\
\hline OsMlo- 6 & 0.600 & 0.338 & 0.265 & 0.316 & 0.383 & 0.313 \\
\hline OsMlo-7 & 0.287 & 0.400 & 0.252 & 0.360 & 0.286 & 0.478 \\
\hline OsMlo- 8 & 0.271 & 0.410 & 0.282 & 0.353 & 0.286 & 0.663 \\
\hline OsMlo-9 & 0.196 & 0.472 & 0.209 & 0.295 & 0.198 & 0.266 \\
\hline OsMlo-10 & 0.305 & 0.502 & 0.285 & 0.684 & 0.315 & 0.370 \\
\hline OsMlo-11 & 0.214 & 0.257 & 0.670 & 0.227 & 0.254 & 0.241 \\
\hline OsMlo-12 & 0.282 & 0.232 & 0.188 & 0.206 & 0.323 & 0.212 \\
\hline
\end{tabular}

${ }^{a}$ The values for TaMlo-1 are means of those for the three homoeologous genes. 
the deduced amino acid sequences of the wheat Mlo members identified in this study and those of 2 barley (Devote et al., 2003), 15 Arabidopsis (Chen et al., 2006), 12 rice (Liu and Zhu, 2008), 8 fern (Selaginella moellendorffii), and 10 moss (Physcomitrella patens) Mlo members was performed using CLUSTALW (Thompson et al., 1994). The fern and moss Mlo members were obtained from the files "Selmo1_GeneModels_AllModels_20071019_aa.fasta" and "proteins.Phypa1_1.FilteredModels.fasta.gz", which were downloaded from the JGI ftp site (ftp://ftp.jgi-psf.org/pub/ JGI_data/). They were not annotated, but we identified them as Mlo members by a BLAST search, as described above. The amino acid sequence similarity between each pair of Mlo members was calculated by MEGA4 (Tamura et al., 2007). Based on the amino acid sequence similarity, a neighbor-joining ( $\mathrm{NJ}$ ) phylogenetic tree of the plant Mlo members was constructed using MEGA4 (Tamura et al., 2007). Bootstrap tests were conducted using 1,000 replicates.

\section{RESULTS}

Identification of additional members of the Mlo family in wheat Our in silico search of public databases using rice and Arabidopsis Mlo family members as queries detected a total of 102 wheat sequences that showed significant similarity to the Mlo members of the other plants. However, none of them, except three sequences known as TaMlo-1, had been annotated as Mlo genes. Among the 102 sequences, 26 were identified to be partial sequences of TaMlo-1 (Table 1). Based on their sequence homology, the remaining 76 sequences were assembled into six unified sequences, and we tentatively named them TaMlo-2, 3, 4, 5, 6, and 7. All of the unified sequences coded for almost full-length sequences with partial UTR sequences, except for TaMlo-3 (Table 1). Based on sequence alignment, specific primers for each of the TaMlo genes were designed. To verify the presence of the unified sequences obtained in silico, we conducted RT-PCR using specific primers and determined the (almost) full sequences of the cDNA for all TaMlo genes, except for TaMlo-3. The comparisons with the barley and rice Mlo members are shown in Fig. 1 and Table 2. The determined cDNA sequences of the TaMlo genes were deposited in DDBJ, and the accession numbers are listed in Table 1.

We deduced the amino acid sequences of the TaMlo genes except TaMlo-3 and revealed that all sequences contained seven transmembrane domains, which is a characteristic feature of MLO proteins (Figs. 1 and 2). The amino-acid sequence similarity between the TaMlo members varied from $26.0 \%$ (between TaMlo-1 and
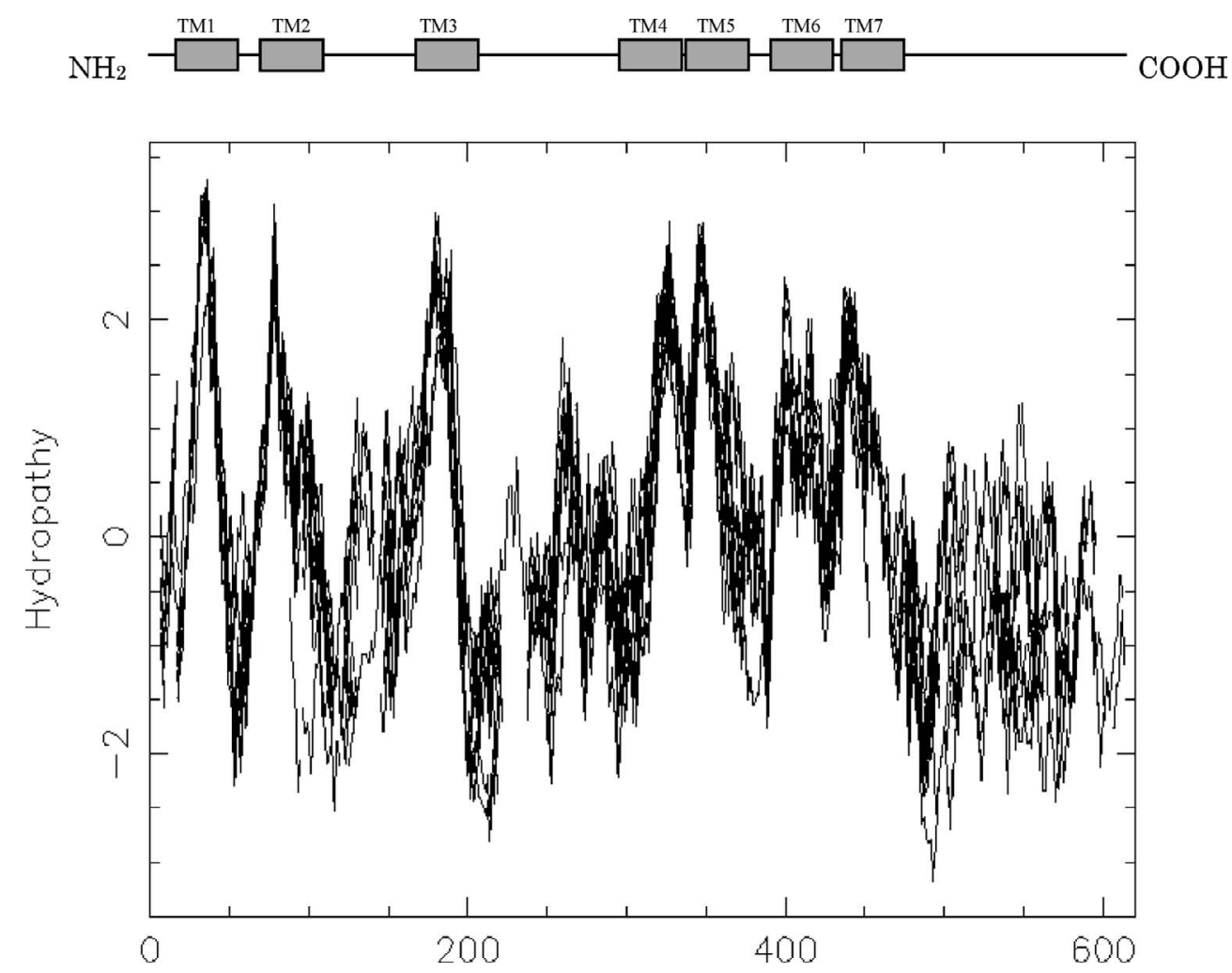

Fig. 2. Hydropathy plotting of the 13 Gramineae Mlo members shown in Fig. 1 obtained using Pepwindowall. The lines almost overlapped, showing the same pattern of hydropathy change. The seven hydropathy peaks in the panel accord with the positions of the seven putative transmembrane regions in TaMLO-1A predicted by HMMTOP shown above. 
TaMlo-4) to $51.6 \%$ (between TaMlo-2 and TaMlo-5) (Table 2). As shown in Fig. 1, the transmembrane domains were relatively conserved among the Mlo members. The level of similarity between TaMlo members was comparable to that between the Mlo members of rice and between those of Arabidopsis. A sequence comparison revealed that WESR3, the gene isolated as a salt-responding gene by Nemoto and Sasakuma (2002), was a partial sequence of TaMlo-2.

Expressional analysis of the TaMlo genes The exp- ression profiles of the TaMlo genes under salt and osmotic stress were investigated by RT-PCR (Fig. 3). TaMlo-1 and TaMlo-2 showed constitutive expression in roots and shoots under both stress and control conditions. TaMlo3 and TaMlo-4 exhibited root-specific expression under both stress and control conditions. Among the TaMlo members, TaMlo-5 showed the highest expression in roots and shoots under both stress and control conditions. TaMlo-6 showed the weakest expression among the TaMlo members, and its expression increased in roots under salt stress and in shoots under osmotic stress (Fig.

(a)
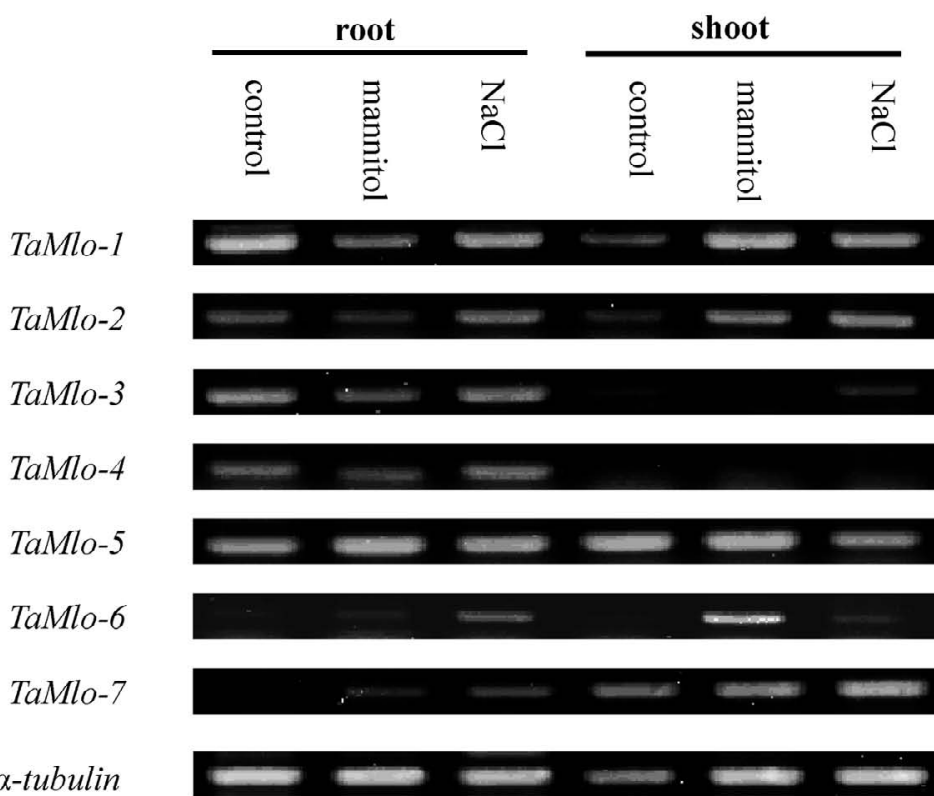

(b)

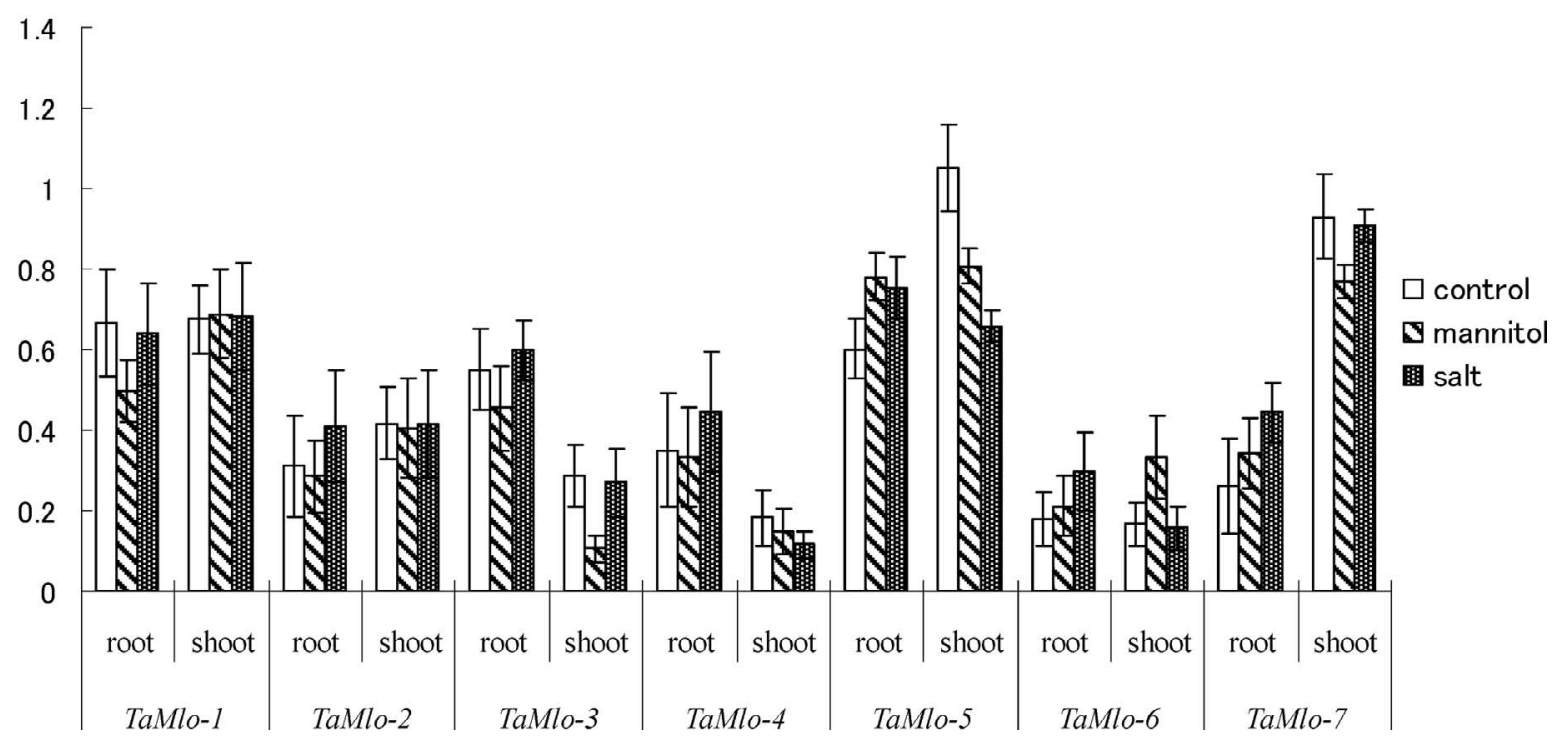

Fig. 3. Expression profiles of the seven TaMlo members in roots and shoots under salt and osmotic stress conditions. (a) Electrophoresis profiles of the RT-PCR products of the TaMlo members and (b) quantification of the expression of TaMlo members by RT-PCR shown as a bar graph with error bars. 


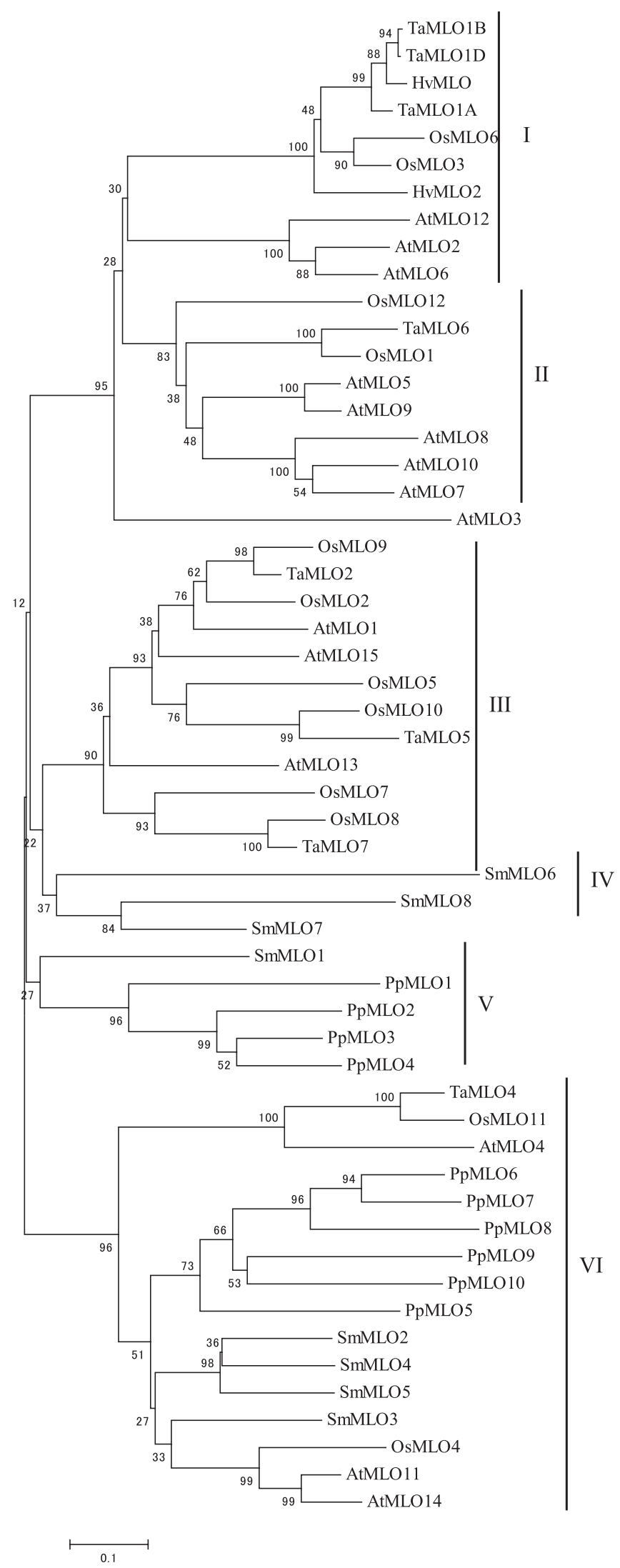

3), although the increase was not significant. TaMlo-7 was predominantly expressed in shoots and demonstrated slightly inducible expression in roots under both salt and osmotic stresses, although the induction level was not statistically significant (Fig. 3). Thus, none of the TaMlo members showed significant increases or decreases in response to stress conditions.

Phylogenetic analysis To clarify the phylogenetic relationships among the Mlo family members of wheat (except TaMlo-3) and other plants, a phylogenetic tree consisting of $55 \mathrm{Mlo}$ members of dicots, monocots, a fern, and a moss was constructed (Fig. 4). In the phylogenetic tree, the members were mainly divided into six major clusters, Classes I - VI. Classes I, II and III consisted of only angiosperm Mlo. Classes IV and V consisted of only fern and moss Mlo. Class VI was composed of various Mlo from all plant species. The four major Mlo clusters reported by Liu and Zhu (2008) correspond to Classes I, II, III, and VI in this study, and each of the major clusters includes Arabidopsis and rice Mlo members.

The seven TaMlo members were classified into four clusters. TaMlo-1, TaMlo-4, and TaMlo- 6 belonged to Class I, Class VI, and Class II, respectively. TaMlo-2, TaMlo-5, and TaMlo-7 were included in Class III, and each had rice orthologs (Fig. 4). Although a partial TaMlo-3 sequence was excluded from the phylogenetic analysis, its sequence homology indicated that TaMlo-3 should be placed in Class III.

\section{DISCUSSION}

Characteristics of wheat Mlo The level of sequence similarity (Table 2) and phylogenetic relationships between the plant Mlo members demonstrated that the seven identified wheat Mlo members were not homoeologous to one another, in spite of the hexaploid nature of wheat. Since Arabidopsis and rice, which are diploid species, possess 15 and 12 Mlo members, respectively, wheat, which has a larger genome than these species, should have more Mlo members. The reason we only identified seven Mlo members in this study is probably because not all Mlo members are expressed constitutively in wheat. Chen et al. (2006) revealed that some of the Mlo members in Arabidopsis are only expressed in single specific organs such as the inflorescence. In this study,

Fig. 4. A phylogenetic tree based on the amino acid sequences of the Mlo members of 55 land plants constructed by the neighbor-joining method. The numbers next to the nodes represent the bootstrap percentages after 1,000 replications. Scale bars are shown below the tree. AtMlos, OsMlos, SmMlos, and PpMlos are the Mlo members obtained from Arabidopsis thaliana, rice (Oryza sativa), fern (Selaginella moellendorffiif), and moss (Physcomitrella patens), respectively. 
we also found organ specific expression of Mlo. It is noteworthy that the seven wheat Mlo members covered the four major groups of angiosperm Mlo. Presumably the Mlo member-specific primers we designed in this study amplified the three homoeologous loci simultaneously.

Evolution of plant Mlo In the phylogenetic tree we produced, the plant Mlo members were divided into six classes; four of them included members of both monocot and dicot plants. This indicated that the formation of Mlo classes preceded the divergence of monocot and dicot plants. In each class, the genetic relationships between the sequences were generally consistent with the phylogeny of the plant species. For example, the wheat Mlo members were closer to the rice $M l o$ members than to the Arabidopsis members in each cluster. In Class VI, the members of three angiosperm species formed a cluster and joined with the cluster of the fern Mlo members, and the moss Mlo members were located as an outgroup of the Mlo members of vascular plants. These results indicated that the Mlo members of each class were generally conserved during the evolution of land plants. However, in view of the relationships of the Gramineae family, there are inconsistencies. In Class I, the barley Mlo gene was placed in a cluster with three homoeologous wheat Mlo members. This result might have been caused by differences in the evolutionary rate among the homoeologous loci. Since the Class VI Mlo were found in the angiosperm, fern, and moss species, they may be regarded as the most conserved group of Mlo with functional importance in land plants. Classes IV and V are specific to ferns and mosses. Ferns and mosses are not a monophyletic group, and therefore, the loss of these classes from the angiosperm lineage is more likely to have occurred than their gain in the fern and moss lineage.

Each class contained various numbers of Mlo members from a single species. For example, Class II had five members from Arabidopsis and two from rice, and Class III included six members from rice and three from Arabidopsis. Four of the seven wheat Mlo members, including the partial sequence of TaMlo-3, belonged to Class III. The relatively larger number of Class III members in wheat, as was found in rice and maize (Liu and $\mathrm{Zhu}, 2008$ ), suggests the occurrence of an increase in Class III members in the monocot or Gramineae lineage.

The barley Mlo members, which are the key genes responsible for powdery mildew resistance (Jørgensen, 1992; Wolter et al., 1993), were included in Class I together with the wheat homologs $T a M l o-1$, as reported by a previous study (Liu and Zhu, 2008). All of the other wheat Mlo members were newly identified in this study and were classified into different classes from the barley Mlo.

Expression of wheat Mlo Based on their expression patterns, the TaMlo members were classified into four types. The first type comprised TaMlo-1, TaMlo-2, and TaMlo-5, which were expressed constitutively in both roots and shoots. The second and the third types consisted of TaMlo-3 and TaMlo-4, which displayed rootspecific expression, and TaMlo-7, which displayed shootspecific expression, respectively. The fourth type comprised TaMlo- 6 and displayed weak expression. In the phylogenetic tree, the seven wheat Mlo members were also classified into four classes, but their expression profiles did not correspond to their phylogenetic relationships. Three Class III TaMlo members, TaMlo-2, TaMlo5, and TaMlo-7, showed different expression patterns from each other, and the two members with root-specific expression, TaMlo-3 and TaMlo-4, were classified into Classes III and V, respectively. Also, in Arabidopsis, the organ-specific expression patterns of the Mlo genes did not correspond to the phylogenetic relationships among the Mlo members (Chen et al., 2006). These facts suggest that differentiation of the expression patterns of $M l o$ classes occurred after they had diverged and that organspecific expression developed independently in the respective species.

As for salt and osmotic stress, neither significant induction nor a significant reduction in the expression of the TaMlo genes was observed in this study. Even TaMlo-2, a homolog of WESR3 that was isolated as an early saltresponding gene in wheat (Nemoto and Sasakuma, 2002), showed no significant expressional change in response to salt or osmotic stress. This suggests that TaMlo(s) responds to salt stress quickly but not continuously, as reported for the salt and osmotic responses of the Mlo genes in Arabidopsis (Chen et al., 2006). In fact, the responses of the Mlo genes to biotic and abiotic stresses are various (early, late, or continuous) and depend on the type of stress; for example, wounding causes a rapid increase in the expression of the Mlo genes in Arabidopsis, whereas fungal infection induces the expression of the Mlo genes five days after infection in Arabidopsis and barley (Jarosch et al., 2003; Chen et al., 2006). These diverse $M l o$ responses make it difficult to understand the mechanism and functional role of the Mlo genes.

In this study, we demonstrated that at least seven members of the Mlo family are present in wheat (actually at least 21 if the hexaploidy of bread wheat is considered), and some of them showed organ-specific expression. The Mlo gene is a key gene for resistance to powdery mildew in barley (Jørgensen, 1992; Wolter et al., 1993). The finding of novel Mlo loci and clarification of their expression profiles will aid future wheat breeding aimed at powdery mildew resistance.

This work was supported by a Grant-in-Aid for Scientific Research (A) (16201047). We express our gratitude to Dr. Yasue Nemoto for providing experimental advice. We thank Dr. 
Toshinori Abe and the members of the Laboratory of Plant Genetics and Breeding, Faculty of Agriculture, Yamagata University, and the Laboratory of Evolutionary Genetics, Kihara Institute for Biological Research, for their kind help and continuous encouragement throughout this study.

\section{REFERENCES}

Büschges, R., Hollricher, K., Panstruga, R., Simons, G., Wolter, M., Frijters, A., van Daelen, R., van der Lee, T., Diergaarde, P., Groenendijk, J., et al. (1997) The barley Mlo gene: a novel control element of plant pathogens resistance. Cell 88, 695-705.

Chen, Z., Hartmann, A. H., Wu, M., Friedman, E. J., Chen, J., Pulley, M., Schulze-Lefert, P., and Panstruga, R. (2006) Expression analysis of the $A t M L O$ gene family encoding plant-specific seven-transmembrane domain proteins. Plant Mol. Biol. 60, 583-597.

Devoto, A., Piffanelli, P., Nilsson, I., Wallin, E., Panstruga, R., von Heijne, G., and Schulze-Lefert, P. (1999) Topology, subcellular localization, and sequence diversity of the Mlo family in plants. J. Biol. Chem. 274, 34993-35004.

Devoto, A., Hartmann, A. H., Piffanelli, P., Elliott, C., Simmons, C., Taramino, G., Goh, C.-S., Cohen, E. F., Emerson, C. B. Schulze-Lefert, P., et al. (2003) Molecular phylogeny and evolution of the plant-specific seven-transmembrane MLO family. J. Mol. Evol. 56, 77-88.

Elliott, C., Zhou, F., Spielmeyer, W., Panstruga, R., and SchulzeLefert, P. (2002) Functional conservation of wheat and rice Mlo orthologs in defense modulation to the powdery mildew fungus. Mol. Plant-Microbe Interact. 15, 1069-1077.

Huang, X., and Madan, A. (1999) CAP3: A DNA sequence assembly program. Genome Res. 9, 868-877.

Jarosch, B., Jansen, M., and Schaffrath, U. (2003) Acquired resistance functions in mlo barley, which is hypersusceptible to Magnaporthe grisea. Mol. Plant-Microbe Interact. 16, 107-114.

Jiang, Y., and Deyholos, M. K. (2006) Comprehensive transcriptional profiling of $\mathrm{NaCl}$-stressed Arabidopsis roots reveals novel classes of responsive genes. BMC Plant Biol. 6, 25.

Jørgensen, J. H. (1992) Discovery, characterization and exploitation of Mlo powdery mildew resistance in barley. Euphytica 63, 141-152.

Kim, M. C., Panstruga, R., Elliott, C., Müller, J., Devoto, A., Yoon, H. W., Park, H., Cho, M. J., and Schulze-Lefert, P. (2002) Calmodulin interacts with MLO to regulate defense against mildew in barley. Nature 416, 447-450.

Kyte, J., and Doolittle, R.F. (1982) A simple method for displaying the hydropathic character of a protein. J. Mol. Biol. 157, 105-132.
Liu, Q., and Zhu, H. (2008) Molecular evolution of the $M L O$ gene family in Oryza sativa and their functional divergence. Gene 409, 1-10.

Nemoto, Y., and Sasakuma, T. (2002) Differential stress responses of early salt-stress responding genes in common wheat. Phytochemistry 61, 129-133.

Peterhänsel, C., Freialdenhoven, A., Kurth, J., Kolsch, R., and Schulze-Lefert, P. (1997) Interaction analyses of genes required for resistance responses to powdery mildew in barley reveal distinct pathways leading to leaf cell death. Plant Cell 9, 1397-1409.

Piffanelli, P., Zhou, F., Casais, C., Orme, J., Scharath, U., Collins, N., Panstruga, R., and Schulze-Lefert, P. (2002) The barley MLO modulator of defence and cell death is responsive to biotic and abiotic stress stimuli. Plant Physiol. 129, 1076-1085.

Piffanelli, P., Ramsay, L., Waugh, R., Benabdelmouna, A., D’Hont, A., Hollricher, K., Jørgensen, J. H., Schulze-Lefert, P., and Panstruga, R. (2004) A barley cultivation-associated polymorphism conveys resistance to powdery mildew. Nature 430, 887-891.

Reinstädler, A., Müller, J., Czembor, J. H., Piffanelli, P., and Panstruga, R. (2010) Novel induced mlo mutant alleles in combination with site-directed mutagenesis reveal functionally important domains in the heptahelical barley Mlo protein. BMC Plant Biol. 10, 31.

Tacconi, G., Baldassarre, V., Collins, N. C., Bulgarelli, D., Stanca, A. M., and Vale, G. (2006) Haplotype characterization and markers at the barley Mlo powdery mildew resistance locus as tools for marker-assisted selection. Genome 49, 864-872.

Tamura, K., Dudley, J., Nei, M., and Kumar, S. (2007) MEGA4: Molecular Evolutionary Genetics Analysis (MEGA) software version 4.0. Mol. Biol. Evol. 24, 1596-1599.

Thompson, J. D., Higgins, D. G., and Gibson, T. J. (1994) CLUSTAL W: improving the sensitivity of progressive multiple sequence alignment through sequence weighting, position-specific gap penalties and weight matrix choice. Nucleic Acids Res. 11, 4673-4680.

Tusnády, G. E., and Simon, I. (2001) The HMMTOP transmembrane topology prediction server. Bioinformatics 17, 849850.

Wolter, M., Hollricher, K., Salamini, F., and Schulze-Lefert, P. (1993) The mlo resistance alleles to powdery mildew infection in barley trigger a developmentally controlled defense mimic phenotype. Mol. Gen. Genet. 239, 122-128.

Zellerhoff, N., Himmelbach, A., Dong, W., Bieri, S., Schaffrath, U., and Schweizer, P. (2010) Nonhost resistance of barley to different fungal pathogens is associated with largely distinct, quantitative transcriptional responses. Plant Physiol. 152, 2053-2066. 